\title{
Research Article \\ Fixed Point Problems in Cone Rectangular Metric Spaces with Applications
}

\author{
Muhammad Nazam $\mathbb{D}^{1},{ }^{1}$ Anam Arif, ${ }^{2}$ Hasan Mahmood, ${ }^{2}$ and Sang Og Kim $\mathbb{D}^{3}$ \\ ${ }^{1}$ Department of Mathematics, Allama Iqbal Open University, H-8, Islamabad 44000, Pakistan \\ ${ }^{2}$ Department of Mathematics, Government College University, Lahore, Pakistan \\ ${ }^{3}$ School of Data Science, Hallym University, Chuncheon 24252, Republic of Korea \\ Correspondence should be addressed to Muhammad Nazam; muhammad.nazam@aiou.edu.pk \\ and Sang Og Kim; sokim@hallym.ac.kr
}

Received 30 June 2020; Revised 20 August 2020; Accepted 27 August 2020; Published 10 September 2020

Academic Editor: Bipan Hazarika

Copyright (C) 2020 Muhammad Nazam et al. This is an open access article distributed under the Creative Commons Attribution License, which permits unrestricted use, distribution, and reproduction in any medium, provided the original work is properly cited.

\begin{abstract}
In this paper, we introduce an ordered implicit relation and investigate some new fixed point theorems in a cone rectangular metric space subject to this relation. Some examples are presented as illustrations. We obtain a homotopy result as an application. Our results generalize and extend several fixed point results in literature.
\end{abstract}

\section{Introduction}

Many authors generalized the classical concept of metric space, by changing the metric conditions partially. Branciari [1] introduced rectangular metric space (RMS), where the triangular inequality condition of metric space was replaced by rectangular inequality. He also proved an analog of the Banach contraction principle in rectangular metric spaces. Azam and Arshad [2] mentioned some necessary conditions to get a unique fixed point for Kannan-type mappings in this context. Later, Karapinar et al. [3] investigated some fixed points for $(\psi, \phi)$ contractions on rectangular metric spaces. On the other hand, Di Bari and Vetro [4] used $(\psi, \phi)$ -weakly contractive condition to give an extension of the results in [3]. Subsequently, a number of authors were engrossed in rectangular metric spaces and proved the existence and uniqueness of fixed point theorems for certain types of mappings [2-6].

The significance of the Banach contraction principle lies in the fact that it is a very essential tool to check the existence of solutions for differential equations, integral equations, matrix equations, and functional equations made by mathematical models of real-world problems. There has been a ten- dency for consistent theorists to improve both the underlying space and the contractive condition (explicit type) used by Banach [7] under the effect of one of the structures like order metric structure $[8,9]$, graphic metric structure $[10,11]$, multivalued mapping structure [12-14], $\alpha$-admissible mapping structure [15], comparison functions, and auxiliary functions. The process of developing new fixed point theorems in the complete metric spaces is in progress under various new restrictions. In this regard, we can find very nice results by Debnath et al. that appeared in $[10,12,14]$.

Later on, Popa [16] introduced self-mappings satisfying implicit relation and obtained fixed points, under the effect of these functions. Popa [16-18] obtained some fixed point theorems in metric spaces. However, scrutiny into the fixed points of self-mappings satisfying implicit relations in order metric structure was made by Beg and Butt $[19,20]$, and some common fixed point theorems were established by Berinde and Vetro [21, 22] and Sedghi et al. [23]. Huang and Zhang [24] introduced cone metric by replacing real numbers with ordering Banach spaces and established a convergence criterion for sequences in cone metric space to generalize Banach fixed point theorem. Huang and Zhang [24] considered the concept of normal cone for their drawn 
outcomes; however, Rezapour and Hamlbarani [25] left the normality condition in some results by Huang. Many authors have investigated fixed point theorems and common fixed point theorems of self-mappings for normal and nonnormal cones in cone metric spaces (see [26-29]).

Azam et al. [5] introduced the notion of a cone rectangular metric space and proved the Banach contraction principle in this context. In 2012, Rashwan [6] extended this idea as a continuation, which improved the results in [5]. The appealing nature of these spaces has enticed scrutiny into fixed point theorems for various contractions on cone rectangular metric spaces (see $[5,6])$.

In this paper, we continue the study initiated by Azam et al. [5] subject to an ordered implicit relation. Since every cone metric space is a cone rectangular metric space but not conversely, therefore we prefer to establish results in cone rectangular metric spaces. These results are supported by some examples and an application in homotopy theory.

\section{Preliminaries}

Definition 1. A binary relation $\mathscr{R}$ over a set $\mathbb{Y} \neq \phi$ defines a partial order if $\mathscr{R}$ has the following axioms:

(1) reflexive

(2) antisymmetric

(3) transitive

A set having partial order $\mathscr{R}$ is known as a partially ordered set denoted by $(\mathbb{Y}, \mathscr{R})$.

In the present article, $\mathscr{E}$ stands for a real Banach space. Now, we present some definitions and relevant results, which will be required in the sequel.

Definition 2 (see [24]). A subset $\mathscr{P}$ of $\mathscr{E}$ is called a cone if and only if the following conditions are satisfied:

(1) $\mathscr{P}$ is closed, nonempty, and $\mathscr{P} \neq\{0\}$

(2) $\alpha \sigma+\beta \varsigma \in \mathscr{P}$, for all $\sigma, \varsigma \in \mathscr{P}$ and $\alpha, \beta \in \mathbb{R}$ such that $\alpha, \beta \geq 0$

(3) $\mathscr{P} \cap(-\mathscr{P})=\{0\}$

Given $\mathscr{P} \subset \mathscr{E}$, define the partial order $\preceq$ with respect to $\mathscr{P}$ as follows:

$\sigma \leq \varsigma$ if and only if $\varsigma-\sigma \in \mathscr{P}$ for all $\sigma, \varsigma \in \mathscr{E}$.(5)

$\sigma \prec \varsigma$ represents that $\sigma \preceq \varsigma$ but $\sigma \neq \varsigma$, while $\sigma \ll \varsigma$ stands for $\varsigma-\sigma \in \mathscr{P}^{\circ}$ (interior of $\mathscr{P}$ ).

Definition 3 (see [24]). The cone $\mathscr{P} \subseteq \mathscr{E}$ is called normal if, for all $\sigma, \varsigma \in \mathscr{P}$, there exists $\mathscr{K}>0$ such that

$$
0 \preceq \sigma \preceq \varsigma \Rightarrow\|\sigma\| \leq \mathscr{K}\|\varsigma\|
$$

Throughout this paper, we assume $\mathbb{Y}=(\mathbb{Y}, \mathscr{R})$ and $\preceq$ a partial order with respect to the cone $\mathscr{P}$ defined in $\mathscr{E}$. If $\mathbb{Y}$ $\subseteq \mathscr{E}$, then $\mathscr{R}$ and $\preceq$ are identical; otherwise, they are different.
Definition 4 (see [24]). Let $\mathbb{Y}$ be a nonempty set, and $d_{c}: \mathbb{Y}$ $\times \mathbb{\mathscr { E }}$ satisfies the following:

(d1) $d_{c}(\sigma, \varsigma) \geq 0, \forall \sigma, \varsigma \in \mathbb{Y}$ and $d_{c}(\sigma, \varsigma)=0$ if and only if $\sigma=\varsigma$

(d2) $d_{c}(\sigma, \varsigma)=d_{c}(\varsigma, \sigma)$

(d3) $d_{c}(\sigma, \xi) \preceq d_{c}(\sigma, \varsigma)+d_{c}(\varsigma, \xi), \forall \sigma, \varsigma, \xi \in \mathbb{Y}$

Then, $d_{c}$ is called a cone metric on $\mathbb{Y}$, and $\left(\mathbb{Y}, d_{c}\right)$ is then known as a cone metric space.

Example 1 (see [5]). Let $\mathbb{Y}=\mathbb{R}, \mathscr{E}=\mathbb{R}^{2}$, and $\mathscr{P}=\{(\sigma, \varsigma) \in$ $\mathscr{E}: \sigma, \varsigma \geq 0\} \subset \mathbb{R}^{2}$. Define $d_{c}: \mathbb{Y} \times \mathbb{Y} \rightarrow \mathscr{E}$ by

$$
d_{c}(\sigma, \varsigma)=(|\sigma-\varsigma|, \alpha|\sigma-\varsigma|)
$$

where $\alpha \geq 0$ is a constant. Then, $d_{c}$ defines a cone metric on $\mathbb{Y}$ Proposition 5 (see [5]). Consider a cone metric space $\left(\mathbb{Y}, d_{c}\right)$, with cone $\mathscr{P}$. Then, for $u, v, w \in \mathscr{E}$, we have

(1) If $u \preceq \alpha u$ and $\alpha \in[0,1)$, then $u=0$

(2) If $0 \preceq u \ll v$ for each $0 \ll v$, then $u=0$

(3) If $u \preceq v$ and $v \ll w$, then $u \ll w$

Surely, cone metric space "being space" generalizes metric space, because in cone metric space, the range of a metric function is an ordered vector space instead of real numbers. Although the set of real numbers is an ordered vector space, we can find many significant ordered vector spaces in the literature (see $[25,27,28,30])$. In Theorem 1.4 and Lemma 2.1 that appeared in $[31,32]$, respectively, the authors developed a metric depending on a given cone metric and proved that a complete cone metric space is always a complete metric space, and then, this relationship between metric and cone metric led them to say that every contraction mapping in a cone metric space is essentially contraction mapping in a metric space.

This paper addresses the fixed point results in the cone rectangular metric spaces. We know that every metric is a rectangular metric but rectangular metric needs not to be a metric (see $[1-3,33]$ ). Also, we know that every cone metric is a cone rectangular metric but conversely does not hold in general (see $[5,6,29])$. In view of observations given in [5, 29 ], we infer that results in this paper are independent of what authors investigated in $[31,32]$. The implicit relation and hence the contractive condition employed are even new and original in the rectangular metric space. The theorems in this paper are new in rectangular metric space, but we choose the cone rectangular metric space for the sake of the generality of our results.

Definition 6 (see [24]). Let A mapping $d_{c r}: \mathbb{Y} \times \mathbb{Y} \mapsto \mathscr{E}$ is said to be a cone rectangular metric if for all $\sigma, \varsigma, \xi, v \in \mathbb{Y}$ the following conditions are satisfied:

(dR1) $0 \preceq d_{c r}(\sigma, \varsigma)$ and $d_{c r}(\sigma, \varsigma)=0$ if and only if $\sigma=\varsigma$

$(\mathrm{dR} 2) d_{c r}(\sigma, \varsigma)=d_{c r}(\varsigma, \sigma)$ 
$(\mathrm{dR} 3) d_{c r}(\sigma, v) \leq d_{c r}(\sigma, \varsigma)+d_{c r}(\varsigma, \xi)+d_{c r}(\xi, v)$ for all distinct $\varsigma, \xi \in \mathbb{Y} \backslash\{\sigma, v\}$

The cone rectangular metric space is denoted by $\left(\mathbb{Y}, d_{c r}\right)$.

Example 2 (see [5]). Let $\mathscr{E}=\mathbb{R}^{2}, \mathscr{P}=\{(\sigma, \varsigma) \in \mathscr{E}: \sigma, \varsigma \geq 0\}$ $\subset \mathbb{R}^{2}, \mathbb{Y}=\mathbb{R}$. Take $\beta>0$, and define

$d_{c r}(\sigma, \varsigma)= \begin{cases}(0,0) & \text { if } \sigma=\varsigma, \\ (3 \beta, 3) & \text { if } \sigma, \varsigma \in\{1,2\}, \sigma \neq \varsigma, \\ (\beta, 1) & \text { if } \sigma \text { and } \varsigma \text { cannot be both at a time in }\{1,2\}, \sigma \neq \varsigma .\end{cases}$

One can easily check that $\left(\mathbb{Y}, d_{c r}\right)$ is a cone rectangular metric space, but not a cone metric space, since $d_{c r}(1,2)=($ $3 \beta, 3)>d_{c r}(1,3)+d_{c r}(3,2)=(2 \beta, 2)$.

Definition 7 (see [1]). Let $\mathscr{E}$ be a real Banach space, $\left(\mathbb{Y}, d_{c r}\right)$ be a cone rectangular metric space and $c \in \mathscr{E}$ with $0 \ll c$.

(1) A sequence $\left\{\sigma_{n}\right\}$ in $\left(\mathbb{Y}, d_{c r}\right)$ is called a Cauchy sequence, if there exists a natural number $N \in \mathbb{N}$ such that $d_{c r}\left(\sigma_{n}, \sigma_{m}\right) \ll c$ for all $n, m>N$

(2) The sequence $\left\{\sigma_{n}\right\}$ is said to be convergent if there exists an $N \in \mathbb{N}$ such that $d_{c r}\left(\sigma_{n}, \sigma\right) \ll c$ for all $n \geq$ $N$ and $\sigma \in \mathbb{Y}$

(3) The $\left(\mathbb{Y}, d_{c r}\right)$ is called complete if every Cauchy sequence converges in $\mathbb{Y}$

\section{Ordered Implicit Relations}

Many authors have used implicit relations to establish fixed point results and have applied these results to solve nonlinear functional equations (see [19-22, 34, 35]).

In this section, we define a new ordered implicit relation and explain it with an example. In the next section, we use it along with some other assumptions to develop some new fixed point theorems in the cone rectangular metric space.

Let $(\mathscr{E},\|\|$.$) be a real Banach space and B(\mathscr{E}, \mathscr{E})$ be the space of all bounded linear operators $T: \mathscr{E} \rightarrow \mathscr{E}$ with the usual norm $\|\cdot\|_{1}$ defined in $B(\mathscr{E}, \mathscr{E})$ that is $\|T\|_{1}=\sup _{v \in \mathscr{E}}(\|$ $T(v)\|/\| v \|) v \neq 0$.

In this section, generalizing the idea of [16], we define the following notion:

Let $\mathscr{L}: \mathscr{E}^{6} \rightarrow \mathscr{E}$ be a continuous operator which satisfies the conditions given below:

$\left(\mathscr{L}_{1}\right) v_{5} \preceq v_{5}$ and $v_{6} \preceq v_{6} \Rightarrow \mathscr{L}\left(v_{1}, v_{2}, v_{3}, v_{4}, v_{5}, v_{6}\right) \preceq \mathscr{L}\left(v_{1}\right.$, $\left.v_{2}, v_{3}, v_{4}, v_{5}, v_{6}\right)$

$\left(\mathscr{L}_{2}\right)$ If $\mathscr{L}\left(v_{1}, v_{2}, v_{2}, v_{1}, v_{1}+v_{2}+v_{3}, v_{1}\right) \preceq 0$, then there exists an order preserving operator $S \in B(\mathscr{E}, \mathscr{E})$ with $\|S\|_{1}<$ 1 such that $v_{1} \preceq S\left(v_{2}\right)$ and $v_{3} \preceq S\left(v_{1}\right)$ for all $v_{1}, v_{2}, v_{3} \in \mathscr{E}$ or if $\mathscr{L}\left(v_{1}, v_{2}, v_{1}, v_{2}, v_{1}, v_{1}+v_{2}+v_{3}\right) \preceq 0$, then $v_{2} \preceq S\left(v_{3}\right)$ and $v_{1} \preceq S($ $v_{2}$ ) for all $v_{1}, v_{2}, v_{3} \in \mathscr{E}$

$\left(\mathscr{L}_{3}\right) \mathscr{L}(v, 0,0, v, v, 0)>0$ whenever $\|v\|>0$

Let $\mathscr{G}=\left\{\mathscr{L}: \mathscr{E}^{6} \rightarrow \mathscr{E} \mid \mathscr{L}\right.$ satisfies conditions $\mathscr{L}_{1}, \mathscr{L}_{2}$, $\left.\mathscr{L}_{3}\right\}$.
Example 3. Let $\preceq$ be the partial order with respect to cone $\mathscr{P}$ as defined in Section 2 and let $(\mathscr{E},\|\|$.$) be a real Banach space. For$ all $v_{i} \in \mathscr{E}(i=1$ to 6$), \alpha \in(0,1 / 3)$ and $(1+\alpha) / 2 \leq \beta \leq 1+\alpha$, define $\mathscr{L}: \mathscr{E}^{6} \rightarrow \mathscr{E}$, by

$$
\mathscr{L}\left(v_{1}, v_{2}, v_{3}, v_{4}, v_{5}, v_{6}\right)=\alpha\left\{v_{2}+v_{3}+v_{4}\right\}-\beta\left(v_{5}+v_{6}\right)+v_{1} .
$$

Then, the operator $\mathscr{L} \in \mathscr{G}$ :

$\left(\mathscr{L}_{1}\right)$ Let $v_{5} \preceq \gamma_{5}$ and $v_{6} \preceq \gamma_{6}$, then $\gamma_{5}-v_{5} \in \mathscr{P}$ and $\gamma_{6}-v_{6}$ $\in \mathscr{P}$. Now, we show that $\mathscr{L}\left(v_{1}, v_{2}, v_{3}, v_{4}, v_{5}, v_{6}\right)-\mathscr{L}\left(v_{1}, v_{2}\right.$, $\left.v_{3}, v_{4}, \gamma_{5}, \gamma_{6}\right) \in \mathscr{P}$. Given that $\gamma_{5}-v_{5} \in \mathscr{P}$ and $\gamma_{6}-v_{6} \in \mathscr{P}$ and by Definition 2 (2), we have

$$
\begin{gathered}
\mathscr{L}\left(v_{1}, v_{2}, v_{3}, v_{4}, v_{5}, v_{6}\right)-\mathscr{L}\left(v_{1}, v_{2}, v_{3}, v_{4}, \gamma_{5}, \gamma_{6}\right) \\
=\alpha\left\{v_{2}+v_{3}+v_{4}\right\}-\beta\left(v_{5}+v_{6}\right)+v_{1}-\left(\alpha\left\{v_{2}+v_{3}+v_{4}\right\}\right. \\
\left.-\beta\left(\gamma_{5}+\gamma_{6}\right)+v_{1}\right)=\beta\left(\gamma_{5}-v_{5}+\gamma_{6}-v_{6}\right) \in \mathscr{P} .
\end{gathered}
$$

Thus, $\mathscr{L}\left(v_{1}, v_{2}, v_{3}, v_{4}, \gamma_{5}, \gamma_{6}\right) \preceq \mathscr{L}\left(v_{1}, v_{2}, v_{3}, v_{4}, v_{5}, v_{6}\right)$.

$\left(\mathscr{L}_{2}\right)$ Let $v_{1}, v_{2}, v_{3} \in \mathscr{E}$ be such that $0 \preceq v_{1}, 0 \preceq v_{2}, 0 \preceq v_{3}$. If $\mathscr{L}\left(v_{1}, v_{2}, v_{2}, v_{1}, v_{1}+v_{2}+v_{3}, v_{1}\right) \preceq 0$ then, we have $-v_{1}-\alpha\left\{v_{2}\right.$ $\left.+v_{2}+v_{1}\right\}+\beta\left(v_{1}+v_{2}+v_{3}+v_{1}\right) \in \mathscr{P}$.

So $(2 \beta-\alpha-1) v_{1}+(\beta-2 \alpha) v_{2}+\beta v_{3} \in \mathscr{P}$.

By Definition 2 (2), we have for $2 \beta \geq \alpha+1$ either

$$
(2 \beta-\alpha-1) v_{1}+(\beta-2 \alpha) v_{2} \in \mathscr{P}
$$

or

$$
(2 \beta-\alpha-1) v_{1}+\beta v_{3} \in \mathscr{P}
$$

or

$$
(\beta-2 \alpha) v_{2}+\beta v_{3} \in \mathscr{P}
$$

For (6), if $v_{1}=0$ and $v_{2} \neq 0$, then $(\beta-2 \alpha) v_{2} \in \mathscr{P}$. Thus, there exists $S: \mathscr{E} \rightarrow \mathscr{E}$ defined by $S\left(v_{2}\right)=\eta v_{2} \quad(\eta=(\beta-2 \alpha)$ is a scalar) such that $v_{1} \preceq S\left(v_{2}\right)$. Now, if $v_{2}=0$ and $v_{1} \neq 0$, then, $(2 \beta-1-\alpha) v_{1} \in \mathscr{P}$. So, there exists $S: \mathscr{E} \rightarrow \mathscr{E}$ defined by $S($ $\left.v_{1}\right)=\eta v_{1}\left(\eta=(2 \beta-1-\alpha)\right.$ is a scalar) such that $v_{2} \preceq S\left(v_{1}\right)$, for some $\alpha \in(0,1 / 3)$. For if both $v_{1} \neq 0$ and $v_{2} \neq 0$, then we get an absurdity.

For (7), if $v_{3}=0$ and $v_{1} \neq 0$, then $(2 \beta-\alpha-1) v_{1} \in \mathscr{P}$. Thus, there exists $S: \mathscr{E} \rightarrow \mathscr{E}$ defined by $S\left(v_{1}\right)=\eta v_{1}$ $\left(\eta=(2 \beta-\alpha-1)\right.$ is a scalar) such that $v_{3} \preceq S\left(v_{1}\right)$. Now, if $v_{3}$ $\neq 0$ and $v_{1}=0$, then, $\beta v_{3} \in \mathscr{P}$. So, there exists $S: \mathscr{E} \rightarrow \mathscr{E}$ defined by $S\left(v_{3}\right)=\eta v_{3}\left(\eta=\beta\right.$ is a scalar) such that $v_{1} \preceq S\left(v_{3}\right)$. For if both $v_{1} \neq 0$ and $v_{3} \neq 0$, then we get an absurdity. Similar arguments hold for (8).

$\left(\mathscr{L}_{3}\right)$ Let $v \in \mathscr{E}$ be such that $\|v\|>0$ and consider, $0 \preceq \mathscr{L}$ $(v, 0,0, v, v, 0)$ then $(1+\alpha-\beta) v \in \mathscr{P}$, which holds whenever $\|v\|>0$.

Similarly, the operators $\mathscr{L}: \mathscr{E}^{6} \rightarrow \mathscr{E}$ defined by

(1) $\mathscr{L}\left(v_{1}, v_{2}, v_{3}, v_{4}, v_{5}, v_{6}\right)=v_{1}-\alpha\left\{v_{5}+v_{6}\right\} ; \alpha \in[1, \infty)$ 
(2) $\mathscr{L}\left(v_{1}, v_{2}, v_{3}, v_{4}, v_{5}, v_{6}\right)=v_{3}-\alpha v_{5}-\beta v_{6} ; \alpha+\beta<1, \alpha$, $\beta>0$

(3) $\mathscr{L}\left(v_{1}, v_{2}, v_{3}, v_{4}, v_{5}, v_{6}\right)=v_{1}-\alpha\left\{v_{3}+v_{4}\right\}-(1-\alpha) \beta$ $\max \left\{v_{5}, v_{6}\right\} ; \alpha \in[0,1)$ and $\beta \in[1 / 2,1)$

(4) $\mathscr{L}\left(v_{1}, v_{2}, v_{3}, v_{4}, v_{5}, v_{6}\right)=v_{1}-\alpha v_{2}$ for some $\alpha \in[0,1)$

(5) $\mathscr{L}\left(v_{1}, v_{2}, v_{3}, v_{4}, v_{5}, v_{6}\right)=v_{1}-k\left(v_{3}+v_{4}\right)$ for some $k \in[$ $0,1 / 2)$ are members of $\mathscr{G}$

The following remark is essential in the sequel.

Remark 8. If $S \in B(\mathscr{E}, \mathscr{E})$, the Neumann series $I+S+S^{2}+\cdots$ $+S^{n}+\cdots$ converges if $\|S\|_{1}<1$ and diverges otherwise. Also, if $\|S\|_{1}<1$, then there exists $\lambda>0$ such that $\|S\|_{1}<\lambda<1$ and $\|$ $S^{n} \|_{1} \leq \lambda^{n}<1$.

\section{New Results}

Recently, Popa [16] has employed implicit type contractive condition on self-mapping to obtain some fixed point theorems. Ran and Reurings [9] have presented an analog of Banach fixed point theorem for monotone self-mappings in an ordered metric space. Huang and Zhang [24] introduced the idea of cone metric spaces and obtained analogs of Banach fixed point theorem, Kannan fixed point theorem, and Chatterjea fixed point theorem in cone metric spaces. In this section, we prove some fixed point results for ordered implicit relations in a cone rectangular metric space which improves the results in $[9,16,24]$. We derive these results under two different partial orders: one defined in underlying set and the other in real Banach space.

Theorem 9. Let $\left(\mathbb{Y}, d_{c r}\right)$ be a complete cone rectangular metric space and $\mathscr{P} \subset \mathscr{E}$ be a cone. Let $f: \mathbb{Y} \rightarrow \mathbb{Y}$. If there exist $T \in$ $B(\mathscr{E}, \mathscr{E})$, identity operator $I: \mathscr{E} \rightarrow \mathscr{E}$ and $\mathscr{L} \in \mathscr{G}$ such that, for all comparable elements $\sigma, \kappa \in \mathbb{Y}$

$$
\begin{aligned}
& (I-T)^{2}(I+T)\left(d_{c r}(\sigma, f(\sigma))\right) \leq d_{c r}(\sigma, \kappa) \text { implies } \mathscr{L} \\
& \quad \cdot\left(d_{c r}(f(\sigma), f(\kappa)), d_{c r}(\sigma, \kappa), d_{c r}(\sigma, f(\sigma)), d_{c r}(\kappa, f(\kappa)), d_{c r}\right. \\
& \left.\quad \cdot\left(\sigma, f^{2}(\kappa)\right), d_{c r}\left(\kappa, f^{2}(\sigma)\right)\right) \preceq 0
\end{aligned}
$$

and

(1) there exists $\sigma_{0} \in \mathbb{Y}$ such that $\sigma_{0} \mathscr{R} f\left(\sigma_{0}\right)$

(2) for all $\sigma, \kappa \in \mathbb{Y}, \sigma \mathscr{R} \kappa$ implies $f(\sigma) \mathscr{R} f(\kappa)$

(3) for every $\left\{\sigma_{n}\right\} \subseteq \mathbb{Y}, d_{c r}\left(\sigma_{n}, \sigma_{n+1}\right) \preceq T\left(d_{c r}\left(\sigma_{n-1}, \sigma_{n}\right)\right)$

(4) for a sequence $\left\{\sigma_{n}\right\}$ with $\sigma_{n} \rightarrow x^{*}$ whose all sequential terms are comparable, we have $\sigma_{n} \mathscr{R} x^{*}$ for all $n \in \mathbb{N}$ and $d_{c r}\left(x^{*}, f\left(x^{*}\right)\right) \preceq d_{c r}\left(x^{*}, f^{2}\left(x^{*}\right)\right)$

Then, $x^{*}=f\left(x^{*}\right)$.
Proof. Let $\sigma_{0} \in \mathbb{Y}$ be as assumed in (1). We construct a sequence $\left\{\sigma_{n}\right\}$ by $f\left(\sigma_{n-1}\right)=\sigma_{n}$ starting with $\sigma_{0} \in \mathbb{Y}$. Then, $\sigma_{0} \mathscr{R} \sigma_{1}$. By assumption (2), we have $\sigma_{1} \mathscr{R} \sigma_{2}, \sigma_{2} \mathscr{R} \sigma_{3} \ldots$ $\sigma_{n-1} \mathscr{R} \sigma_{n}$. For $\sigma=\sigma_{0}$ and $\kappa=\sigma_{1}$, we have by (9).

$$
\begin{aligned}
(I-T)^{2}(I+T)\left(d_{c r}\left(\sigma_{0}, f\left(\sigma_{0}\right)\right)\right) \\
=(I-T)^{2}(I+T)\left(d_{c r}\left(\sigma_{0}, \sigma_{1}\right)\right) \leq d_{c r}\left(\sigma_{0}, \sigma_{1}\right) \text { implies } \mathscr{L} \\
\quad \times\left(d_{c r}\left(f\left(\sigma_{0}\right), f\left(\sigma_{1}\right)\right), d_{c r}\left(\sigma_{0}, \sigma_{1}\right), d_{c r}\left(\sigma_{0}, f\left(\sigma_{0}\right)\right), d_{c r}\right. \\
\left.\quad \times\left(\sigma_{1}, f\left(\sigma_{1}\right)\right), d_{c r}\left(\sigma_{0}, f^{2}\left(\sigma_{1}\right)\right), d_{c r}\left(\sigma_{1}, f^{2}\left(\sigma_{0}\right)\right)\right) \preceq 0
\end{aligned}
$$

that is,

$\mathscr{L}\left(d_{c r}\left(\sigma_{1}, \sigma_{2}\right), d_{c r}\left(\sigma_{0}, \sigma_{1}\right), d_{c r}\left(\sigma_{0}, \sigma_{1}\right), d_{c r}\left(\sigma_{1}, \sigma_{2}\right), d_{c r}\left(\sigma_{0}, \sigma_{3}\right), d_{c r}\left(\sigma_{1}, \sigma_{2}\right)\right) \leq 0$.

By (dR3), we have

$$
d_{c r}\left(\sigma_{0}, \sigma_{3}\right) \preceq d_{c r}\left(\sigma_{0}, \sigma_{1}\right)+d_{c r}\left(\sigma_{1}, \sigma_{2}\right)+d_{c r}\left(\sigma_{2}, \sigma_{3}\right)
$$

and so we rewrite (11) employing condition $\left(\mathscr{L}_{1}\right)$ as follows:

$$
\begin{aligned}
& \mathscr{L}\left(d_{c r}\left(\sigma_{1}, \sigma_{2}\right), d_{c r}\left(\sigma_{0}, \sigma_{1}\right), d_{c r}\left(\sigma_{0}, \sigma_{1}\right), d_{c r}\left(\sigma_{1}, \sigma_{2}\right), d_{c r}\left(\sigma_{0}, \sigma_{1}\right)\right. \\
& \left.\quad+d_{c r}\left(\sigma_{1}, \sigma_{2}\right)+d_{c r}\left(\sigma_{2}, \sigma_{3}\right), d_{c r}\left(\sigma_{1}, \sigma_{2}\right)\right) \preceq 0,
\end{aligned}
$$

and thus, by $\left(\mathscr{L}_{2}\right)$, there exists an order preserving operator $S \in B(\mathscr{E}, \mathscr{E})$ with $\|S\|_{1}<1$ such that

$$
\begin{aligned}
& d_{c r}\left(\sigma_{1}, \sigma_{2}\right) \preceq S\left(d_{c r}\left(\sigma_{0}, \sigma_{1}\right)\right), \\
& d_{c r}\left(\sigma_{2}, \sigma_{3}\right) \preceq S\left(d_{c r}\left(\sigma_{1}, \sigma_{2}\right)\right) .
\end{aligned}
$$

Now, put $\sigma=\sigma_{1}$ and $\kappa=\sigma_{2}$ in (9) to have

$$
\begin{aligned}
& (I-T)^{2}(I+T)\left(d_{c r}\left(\sigma_{1}, f\left(\sigma_{1}\right)\right)\right) \\
& =(I-T)^{2}(I+T)\left(d_{c r}\left(\sigma_{1}, \sigma_{2}\right)\right) \preceq d_{c r}\left(\sigma_{1}, \sigma_{2}\right) \text { implies } \mathscr{L} \\
& \quad \times\left(d_{c r}\left(f\left(\sigma_{1}\right), f\left(\sigma_{2}\right)\right), d_{c r}\left(\sigma_{1}, \sigma_{2}\right), d_{c r}\left(\sigma_{1}, f\left(\sigma_{1}\right)\right), d_{c r}\right. \\
& \left.\quad \times\left(\sigma_{2}, f\left(\sigma_{2}\right)\right), d_{c r}\left(\sigma_{1}, f\left(\sigma_{3}\right)\right), d_{c r}\left(\sigma_{2}, f\left(\sigma_{2}\right)\right)\right) \leq 0,
\end{aligned}
$$

that is,

$\mathscr{L}\left(d_{c r}\left(\sigma_{2}, \sigma_{3}\right), d_{c r}\left(\sigma_{1}, \sigma_{2}\right), d_{c r}\left(\sigma_{1}, \sigma_{2}\right), d_{c r}\left(\sigma_{2}, \sigma_{3}\right), d_{c r}\left(\sigma_{1}, \sigma_{4}\right), d_{c r}\left(\sigma_{2}, \sigma_{3}\right)\right) \leq 0$.

By (dR3), we have

$$
d_{c r}\left(\sigma_{1}, \sigma_{4}\right) \preceq d_{c r}\left(\sigma_{1}, \sigma_{2}\right)+d_{c r}\left(\sigma_{2}, \sigma_{3}\right)+d_{c r}\left(\sigma_{3}, \sigma_{4}\right)
$$


and $\left(\mathscr{L}_{1}\right)$ implies

$$
\begin{aligned}
& \mathscr{L}\left(d_{c r}\left(\sigma_{2}, \sigma_{3}\right), d_{c r}\left(\sigma_{1}, \sigma_{2}\right), d_{c r}\left(\sigma_{1}, \sigma_{2}\right), d_{c r}\left(\sigma_{2}, \sigma_{3}\right), d_{c r}\left(\sigma_{1}, \sigma_{2}\right)\right. \\
& \left.\quad+d_{c r}\left(\sigma_{2}, \sigma_{3}\right)+d_{c r}\left(\sigma_{3}, \sigma_{4}\right), d_{c r}\left(\sigma_{2}, \sigma_{3}\right)\right) \preceq 0 .
\end{aligned}
$$

By $\left(\mathscr{L}_{2}\right)$, there exists $S \in B(\mathscr{E}, \mathscr{E})$ with $\|S\|_{1}<1$ such that

$$
d_{c r}\left(\sigma_{3}, \sigma_{4}\right) \preceq S\left(d_{c r}\left(\sigma_{2}, \sigma_{3}\right)\right) \preceq S^{2}\left(d_{c r}\left(\sigma_{1}, \sigma_{2}\right)\right) \preceq S^{3}\left(d_{c r}\left(\sigma_{0}, \sigma_{1}\right)\right) .
$$

By continuing this pattern, we can construct a sequence $\left\{\sigma_{n}\right\}$ such that $\sigma_{n} \mathscr{R} \sigma_{n+1}$ with $\sigma_{n+1}=f\left(\sigma_{n}\right)$, and

$$
\begin{aligned}
(I & -T)^{2}(I+T)\left(d_{c r}\left(\sigma_{n-1}, f\left(\sigma_{n-1}\right)\right)\right) \\
& =(I-T)^{2}(I+T)\left(d_{c r}\left(\sigma_{n-1}, \sigma_{n}\right)\right) \\
& \leq d_{c r}\left(\sigma_{n-1}, \sigma_{n}\right) \text { implies } d_{c r}\left(\sigma_{n}, \sigma_{n+1}\right) \\
& \leq S\left(d_{c r}\left(\sigma_{n-1}, \sigma_{n}\right)\right) \preceq S^{2}\left(d_{c r}\left(\sigma_{n-2}, \sigma_{n-1}\right)\right) \\
& \leq \cdots \preceq S^{n}\left(d_{c r}\left(\sigma_{0}, \sigma_{1}\right)\right) .
\end{aligned}
$$

For $m, n \in \mathbb{N}$ with $m>n$, we have by Remark 8

$$
\begin{aligned}
d_{c r}\left(\sigma_{n}, \sigma_{m}\right) \leq & d_{c r}\left(\sigma_{n}, \sigma_{n+1}\right)+d_{c r}\left(\sigma_{n+1}, \sigma_{n+2}\right)+. \cdots \cdots \\
& +d_{c r}\left(\sigma_{m-1}, \sigma_{m}\right) \\
\leq & S^{n}\left(d_{c r}\left(\sigma_{0}, \sigma_{1}\right)\right)+S^{n+1}\left(d_{c r}\left(\sigma_{0}, \sigma_{1}\right)\right) \\
& +\cdots \cdots+S^{m-1}\left(d_{c r}\left(\sigma_{0}, \sigma_{1}\right)\right) \\
= & \left(S^{n}+S^{n+1}+\cdots+S^{m-1}\right)\left(d_{c r}\left(\sigma_{0}, \sigma_{1}\right)\right), \\
\leq & \left\{S^{n}\left(1+S+\cdots+S^{m-n-1}+\cdots\right)\right\}\left(d_{c r}\left(\sigma_{0}, \sigma_{1}\right)\right) \\
= & \left.\left\{S^{n}(I-S)^{-1}\right)\right\}\left(d_{c r}\left(\sigma_{0}, \sigma_{1}\right)\right) .
\end{aligned}
$$

Since $\|S\|_{1}<1$, so, $S^{n} \rightarrow 0$ as $n \rightarrow \infty$. Thus, $\lim _{n \rightarrow \infty} d_{c r}($ $\left.\sigma_{n}, \sigma_{m}\right)=0$ which implies that $\left\{\sigma_{n}\right\}$ is a Cauchy sequence in $\mathbb{Y}$. Since $\left(\mathbb{Y}, d_{c r}\right)$ is a complete cone rectangular metric space, so, there exists $x^{*} \in \mathbb{Y}$ such that $\sigma_{n} \rightarrow x^{*}$ as $n \rightarrow \infty$. Equivalently, there exists a natural number $N_{2}$ such that

$$
d_{c r}\left(\sigma_{n}, x^{*}\right) \ll c \text { for all } n \geq N_{2}
$$

We claim that

$$
(I-T)^{2}(I+T)\left(d_{c r}\left(\sigma_{n}, f\left(\sigma_{n}\right)\right)\right) \preceq d_{c r}\left(\sigma_{n}, x^{*}\right) .
$$

We assume against our claim that

$$
\begin{gathered}
(I-T)^{2}(I+T)\left(d_{c r}\left(\sigma_{n}, f\left(\sigma_{n}\right)\right)\right)>d_{c r}\left(\sigma_{n}, x^{*}\right),(I-T)^{2}(I+T) \\
\cdot\left(d_{c r}\left(\sigma_{n+2}, f\left(\sigma_{n+2}\right)\right)\right)>d_{c r}\left(\sigma_{n+2}, x^{*}\right) \text { for some } n \in \mathbb{N}
\end{gathered}
$$

By (dR3), (9), and assumption (3), we have

$$
\begin{aligned}
d_{c r} & \left(\sigma_{n}, f\left(\sigma_{n}\right)\right) \leq d_{c r}\left(\sigma_{n}, x^{*}\right)+d_{c r}\left(x^{*}, \sigma_{n+2}\right)+d_{c r} \\
& \cdot\left(\sigma_{n+2}, \sigma_{n+1}\right) \prec(I-T)^{2}(I+T)\left(d_{c r}\left(\sigma_{n}, f\left(\sigma_{n}\right)\right)\right)+(I-T)^{2} \\
& \cdot(I+T)\left(d_{c r}\left(\sigma_{n+2}, f\left(\sigma_{n+2}\right)\right)\right)+T\left(d_{c r}\left(\sigma_{n}, f\left(\sigma_{n}\right)\right)\right) \prec(I-T)^{2} \\
& \cdot(I+T)\left(d_{c r}\left(\sigma_{n}, f\left(\sigma_{n}\right)\right)\right)+(I-T)^{2}(I+T) T^{2} \\
& \cdot\left(d_{c r}\left(\sigma_{n}, f\left(\sigma_{n}\right)\right)\right)+T\left(d_{c r}\left(\sigma_{n}, f\left(\sigma_{n}\right)\right)\right),(I-T) \\
& \cdot\left(d_{c r}\left(\sigma_{n}, f\left(\sigma_{n}\right)\right)\right) \prec(I-T)^{2}(I+T)\left(I+T^{2}\right) \\
& \cdot\left(d_{c r}\left(\sigma_{n}, f\left(\sigma_{n}\right)\right)\right) d_{c r}\left(\sigma_{n}, f\left(\sigma_{n}\right)\right) \prec(I-T)^{-1}(I-T)^{2}(I+T) \\
& \left.\cdot\left(I+T^{2}\right)\left(d_{c r}\left(\sigma_{n}, f\left(\sigma_{n}\right)\right)\right) \prec\left(I-T^{4}\right) d_{c r}\left(\sigma_{n}, f\left(\sigma_{n}\right)\right)\right) .
\end{aligned}
$$

Thus, $T^{4}\left(d_{c r}\left(\sigma_{n}, f\left(\sigma_{n}\right)\right)\right) \prec 0$, which is an absurdity. Hence, for each $n \geq 1$, we have

$$
(I-T)^{2}(I+T)\left(d_{c r}\left(\sigma_{n}, f\left(\sigma_{n}\right)\right)\right) \preceq d_{c r}\left(\sigma_{n}, x^{*}\right) .
$$

Assume that $\left\|d_{c r}\left(x^{*}, f\left(x^{*}\right)\right)\right\|>0$. As $\sigma_{n-1} \mathscr{R} \sigma_{n}$ and by the assumption (4), we have $\sigma_{n} \mathscr{R} x^{*}$ for all $n \in \mathbb{N}$ and then by (9), we get

$$
\mathscr{L}\left(\begin{array}{c}
d_{c r}\left(f\left(\sigma_{n}\right), f\left(x^{*}\right)\right), d_{c r}\left(\sigma_{n}, x^{*}\right), d_{c r}\left(\sigma_{n}, f\left(\sigma_{n}\right)\right), d_{c r}\left(x^{*}, f\left(x^{*}\right)\right), \\
d_{c r}\left(\sigma_{n-1}, f^{2}\left(x^{*}\right)\right), d_{c r}\left(f^{2}\left(\sigma_{n}\right), f\left(\sigma_{n}\right)\right)
\end{array}\right) \preceq 0 .
$$

Letting $n \rightarrow \infty$ and in view of assumption (4) and (26), we have

$$
\begin{aligned}
& \mathscr{L}\left(d_{c r}\left(x^{*}, f\left(x^{*}\right)\right), d_{c r}\left(x^{*}, x^{*}\right), d_{c r}\left(x^{*}, x^{*}\right), d_{c r}\left(x^{*}, f\left(x^{*}\right)\right), d_{c r}\right. \\
& \left.\cdot\left(x^{*}, f^{2}\left(x^{*}\right)\right), d_{c r}\left(x^{*}, x^{*}\right)\right) \preceq 0, \mathscr{L}\left(d_{c r}\left(x^{*}, f\left(x^{*}\right)\right), 0,0, d_{c r}\right. \\
& \left.\cdot\left(x^{*}, f\left(x^{*}\right)\right), d_{c r}\left(x^{*}, f^{2}\left(x^{*}\right)\right), 0\right) \preceq 0 .
\end{aligned}
$$

By $\left(\mathscr{L}_{1}\right)$, we have

$\mathscr{L}\left(d_{c r}\left(x^{*}, f\left(x^{*}\right)\right), 0,0, d_{c r}\left(x^{*}, f\left(x^{*}\right)\right), d_{c r}\left(x^{*}, f\left(x^{*}\right)\right), 0\right) \preceq 0$.

This is a contradiction to $\left(\mathscr{L}_{3}\right)$. Thus, $\left\|d_{\mathrm{cr}}\left(x^{*}, f\left(x^{*}\right)\right)\right\|$ $=0$. Hence, $d_{c r}\left(x^{*}, f\left(x^{*}\right)\right)=0$. It follows from $(\mathrm{dR} 1)$ that $x^{*}$ $=f\left(x^{*}\right)$.

Theorem 10. Let $\left(\mathbb{Y}, d_{c r}\right)$ be a complete cone rectangular metric space and $f$ be a self-mapping on $\mathbb{Y}$. If for all comparable elements $\sigma, \kappa \in \mathbb{Y}$, there exist $T \in B(\mathscr{E}, \mathscr{E})$, identity operator $I: \mathscr{E} \rightarrow \mathscr{E}$ and $\mathscr{L} \in \mathscr{G}$ such that

$$
\begin{gathered}
(I-T)^{2}(I+T)\left(d_{c r}(\sigma, f(\sigma)) \leq d_{c r}(\sigma, \kappa) \text { implies } \mathscr{L}\right. \\
\cdot\left(d_{c r}(f(\sigma), f(\kappa)), d_{c r}(\sigma, \kappa), d_{c r}(\sigma, f(\sigma)), d_{c r}\right. \\
\left.\cdot(\kappa, f(\kappa)), d_{c r}\left(\sigma, f^{2}(\kappa)\right), d_{c r}\left(\kappa, f^{2}(\sigma)\right)\right) \preceq 0,
\end{gathered}
$$

and 
(1) there exists $\sigma_{0} \in \mathbb{Y}$ such that $f\left(\sigma_{0}\right) \mathscr{R} \sigma_{0}$

(2) for any $\sigma, \kappa \in \mathbb{Y}, \sigma \mathscr{R} \kappa$ implies $f(\kappa) \mathscr{R} f(\sigma)$

(3) for every $\left\{\sigma_{n}\right\} \subseteq \mathbb{Y}, d_{c r}\left(\sigma_{n}, \sigma_{n+1}\right) \preceq T\left(d_{c r}\left(\sigma_{n-1}, \sigma_{n}\right)\right)$

(4) for a sequence $\left\{\sigma_{n}\right\}$ with $\sigma_{n} \rightarrow x^{*}$ whose all sequential terms are comparable, we have $\sigma_{n} \mathscr{R} x^{*}$ for all $n \in \mathbb{N}$ and $d_{c r}\left(x^{*}, f\left(x^{*}\right)\right) \leq d_{c r}\left(x^{*}, f^{2}\left(x^{*}\right)\right)$

Then, $f$ has a fixed point in $\mathbb{Y}$.

Proof. Let $\sigma_{0}$ be in $\mathbb{Y}$ as assumed in (1). Define the sequence $\left\{\sigma_{n}\right\}$ by $\sigma_{n}=f\left(\sigma_{n-1}\right)$ for all $n$. Since $\sigma_{1}=f\left(\sigma_{0}\right) \mathscr{R} \sigma_{0}$ and by assumption (2) $\sigma_{1}=f\left(\sigma_{0}\right) \mathscr{R} f\left(\sigma_{1}\right)=\sigma_{2}$, repeated application of assumption (2) gives us either $\sigma_{n} \mathscr{R} \sigma_{n-1}$ or $\sigma_{n-1} \mathscr{R} \sigma_{n}$ for each $n$. By (30), we have for $\sigma=\sigma_{1}$ and $\kappa=\sigma_{0}$

$$
\begin{aligned}
& (I-T)^{2}(I+T)\left(d_{c r}\left(f\left(\sigma_{0}\right), \sigma_{0}\right)=(I-T)^{2}(I+T)\right. \\
& \quad \cdot\left(d_{c r}\left(\sigma_{1}, \sigma_{0}\right)\right) \leq d_{c r}\left(\sigma_{1}, \sigma_{0}\right) \text { implies } \mathscr{L} \\
& \quad \cdot\left(d_{c r}\left(f\left(\sigma_{1}\right), f\left(\sigma_{0}\right)\right), d_{c r}\left(\sigma_{1}, \sigma_{0}\right), d_{c r}\left(\sigma_{1}, f\left(\sigma_{1}\right)\right), d_{c r}\right. \\
& \quad \cdot\left(\sigma_{0}, f\left(\sigma_{0}\right)\right), d_{c r}\left(\sigma_{1}, f^{2}\left(\sigma_{0}\right)\right), d_{c r}\left(\sigma_{0}, f^{2}\left(\sigma_{1}\right)\right) \leq 0 \Rightarrow \mathscr{L} \\
& \quad \cdot\left(d_{c r}\left(\sigma_{1}, \sigma_{2}\right), d_{c r}\left(\sigma_{0}, \sigma_{1}\right), d_{c r}\left(\sigma_{1}, \sigma_{2}\right), d_{c r}\left(\sigma_{0}, \sigma_{1}\right), d_{c r}\right. \\
& \left.\quad \cdot\left(\sigma_{1}, \sigma_{2}\right), d_{c r}\left(\sigma_{0}, \sigma_{3}\right)\right) \leq 0 .
\end{aligned}
$$

By (dR3), we have

$$
d_{c r}\left(\sigma_{0}, \sigma_{3}\right) \leq d_{c r}\left(\sigma_{0}, \sigma_{1}\right)+d_{c r}\left(\sigma_{1}, \sigma_{2}\right)+d_{c r}\left(\sigma_{2}, \sigma_{3}\right)
$$

and then using $\mathscr{L}_{1}$, we obtain

$$
\begin{array}{r}
\mathscr{L}\left(d_{c r}\left(\sigma_{1}, \sigma_{2}\right), d_{c r}\left(\sigma_{0}, \sigma_{1}\right), d_{c r}\left(\sigma_{1}, \sigma_{2}\right), d_{c r}\left(\sigma_{0}, \sigma_{1}\right), d_{c r}\right. \\
\cdot\left(\sigma_{1}, \sigma_{2}\right), d_{c r}\left(\sigma_{0}, \sigma_{1}\right)+d_{c r}\left(\sigma_{1}, \sigma_{2}\right)+d_{c r}\left(\sigma_{2}, \sigma_{3}\right) \preceq 0 .
\end{array}
$$

By $\left(\mathscr{L}_{2}\right)$, there exists an order preserving operator $S \in B$ $(\mathscr{E}, \mathscr{E})$ with $\|S\|_{1}<1$ such that

$$
\begin{aligned}
& d_{c r}\left(\sigma_{1}, \sigma_{2}\right) \leq S\left(d_{c r}\left(\sigma_{0}, \sigma_{1}\right)\right), \\
& d_{c r}\left(\sigma_{2}, \sigma_{3}\right) \leq S\left(d_{c r}\left(\sigma_{1}, \sigma_{2}\right)\right) .
\end{aligned}
$$
have

Using (2)f( $\left.\sigma_{0}\right) \mathscr{R} f\left(\sigma_{1}\right)$, take $\sigma=\sigma_{1}$ and $\kappa=\sigma_{2}$ in (30), we

$$
\begin{aligned}
(I-T)^{2}(I+T)\left(d_{c r}\left(\sigma_{1}, f\left(\sigma_{1}\right)\right)=(I-T)^{2}(I+T)\right. \\
\quad \cdot\left(d_{c r}\left(\sigma_{1}, \sigma_{2}\right)\right) \leq d_{c r}\left(\sigma_{1}, \sigma_{2}\right) \text { implies } \mathscr{L}\left(d_{c r}\left(f\left(\sigma_{1}\right), f\left(\sigma_{2}\right)\right), d_{c r}\right. \\
\quad \cdot\left(\sigma_{1}, \sigma_{2}\right), d_{c r}\left(\sigma_{1}, f\left(\sigma_{1}\right)\right), d_{c r}\left(\sigma_{2}, f\left(\sigma_{2}\right)\right), d_{c r}\left(\sigma_{1}, f^{2}\left(\sigma_{2}\right)\right), d_{c r} \\
\left.\quad \cdot\left(\sigma_{2}, f^{2}\left(\sigma_{1}\right)\right)\right) \leq 0 \Rightarrow \mathscr{L}\left(d_{c r}\left(\sigma_{2}, \sigma_{3}\right), d_{c r}\left(\sigma_{1}, \sigma_{2}\right), d_{c r}\right. \\
\left.\quad \cdot\left(\sigma_{1}, \sigma_{2}\right), d_{c r}\left(\sigma_{2}, \sigma_{3}\right), d_{c r}\left(\sigma_{1}, \sigma_{4}\right), d_{c r}\left(\sigma_{2}, \sigma_{3}\right)\right) \leq 0 .
\end{aligned}
$$

By (dR3), $\left(\mathscr{L}_{1}\right)$ and $\left(\mathscr{L}_{2}\right)$, we get

$d_{c r}\left(\sigma_{3}, \sigma_{4}\right) \leq S\left(d_{c r}\left(\sigma_{2}, \sigma_{3}\right)\right) \preceq S^{2}\left(d_{c r}\left(\sigma_{1}, \sigma_{2}\right)\right) \preceq S^{3}\left(d_{c r}\left(\sigma_{0}, \sigma_{1}\right)\right)$.

By continuing the pattern, we construct a sequence $\left\{\sigma_{n}\right\}$ such that

$d_{c r}\left(\sigma_{n}, \sigma_{n+1}\right) \leq S\left(d_{c r}\left(\sigma_{n-1}, \sigma_{n}\right)\right) \leq S^{2}\left(d_{c r}\left(\sigma_{n-2}, \sigma_{n-1}\right)\right) \leq \cdots \leq S^{n}\left(d_{c r}\left(\sigma_{0}, \sigma_{1}\right)\right)$.

Hence, by the same reasoning as in the proof of Theorem 9 , we have $x^{*}=f\left(x^{*}\right)$.

Theorem 11. Let $\left(\mathbb{Y}, d_{c r}\right)$ be a complete cone rectangular metric space and $f$ be a monotone self-mapping on $\mathbb{Y}$. If for all comparable elements $\sigma, \kappa \in \mathbb{Y}$, there exist $T \in B(\mathscr{E}, \mathscr{E})$, identity operator $I: \mathscr{E} \rightarrow \mathscr{E}$, and $\mathscr{L} \in \mathscr{G}$ such that

$$
\begin{gathered}
(I-T)^{2}(I+T)\left(d_{c r}(\sigma, f(\sigma))\right) \leq d_{c r}(\sigma, \kappa) \text { implies } \mathscr{L} \\
\cdot\left(d_{c r}(f(\sigma), f(\kappa)), d_{c r}(\sigma, \kappa), d_{c r}(\sigma, f(\sigma)), d_{c r}\right. \\
\left.\cdot(\kappa, f(\kappa)), d_{c r}\left(\sigma, f^{2}(\kappa)\right), d_{c r}\left(\kappa, f^{2}(\sigma)\right)\right) \leq 0,
\end{gathered}
$$

and

(1) there exists $\sigma_{0} \in \mathbb{Y}$ such that either $\sigma_{0} \mathscr{R} f\left(\sigma_{0}\right)$ or $f($ $\left.\sigma_{0}\right) \mathscr{R} \sigma_{0}$

(2) for every $\left\{\sigma_{n}\right\} \subseteq \mathbb{Y}, d_{c r}\left(\sigma_{n}, \sigma_{n+1}\right) \preceq T\left(d_{c r}\left(\sigma_{n-1}, \sigma_{n}\right)\right)$

(3) for a sequence $\left\{\sigma_{n}\right\}$ with $\sigma_{n} \rightarrow x^{*}$ whose all sequential terms are comparable, we have $\sigma_{n} \mathscr{R} x^{*}$ for all $n \in \mathbb{N}$ and $d_{c r}\left(x^{*}, f\left(x^{*}\right)\right)^{\circ} d_{c r}\left(x^{*}, f^{2}\left(x^{*}\right)\right)$

Then, $f$ has a fixed point in $\mathbb{Y}$.

Proof. This proof follows the same pattern as given in the previous two proofs, so, we omit it.

Remark 12.

(1) In Theorem 9, Theorem 10, and Theorem 11, uniqueness of the fixed point of $f$ can be attained by assuming that for every pair of elements $\sigma, \kappa \in X$, there exists either an upper bound or lower bound of $\sigma, \kappa$

(2) The cone is taken as nonnormal in the above theorems

Theorem 13. Let $\left(\mathbb{Y}, d_{c r}\right)$ be a complete cone rectangular metric space and $f$ be a monotone self-mapping on $\mathbb{Y}$. If for all comparable elements $\sigma, \kappa \in \mathbb{Y}$, there exist $T \in B(\mathscr{E}, \mathscr{E})$, 
identity operator $I: \mathscr{E} \rightarrow \mathscr{E}$, and $\mathscr{L} \in \mathscr{G}$ such that

$(I-T)^{2}(I+T)\left(d_{c r}(\sigma, f(\sigma))\right) \leq d_{c r}(\sigma, \kappa)$ implies $d_{c r}(f(\sigma), f(\kappa)) \leq S\left(d_{c r}(\sigma, \kappa)\right)$.

Moreover, if

(1) there exists $\sigma \in \mathbb{Y}$ such that $\sigma_{0} \mathscr{R} f\left(\sigma_{0}\right)$ or $f\left(\sigma_{0}\right) \mathscr{R} \sigma_{0}$

(2) for a sequence $\left\{\sigma_{n}\right\}$ with $\sigma_{n} \rightarrow x^{*}$ whose all sequential terms are comparable, we have $\sigma_{n} \mathscr{R} x^{*}$ for all $n \in \mathbb{N}$ and $d_{c r}\left(x^{*}, f\left(x^{*}\right)\right) \leq d_{c r}\left(x^{*}, f^{2}\left(x^{*}\right)\right)$

Then, there exists $x^{*} \in \mathbb{Y}$ such that $x^{*}=f\left(x^{*}\right)$.

Proof. Define $S(v)=q v$ for all $v \in \mathscr{E}$ and $q \in[0,1)$, then $S \in$ $B(\mathscr{E}, \mathscr{E})$ with $\|S\|_{1}<1$ also define implicit relation by

$\mathscr{L}\left(\sigma_{1}, \sigma_{2}, \sigma_{3}, \sigma_{4}, \sigma_{5}, \sigma_{6}\right)=\sigma_{1}-\alpha \sigma_{2}$ forsome $\alpha \in[0,1)$.

The proof follows by the application of Theorem 9 .

The following examples illustrate the main theorem.
Example 4. Let $\mathscr{E}=\left(\mathbb{R}^{3},\|\cdot\|\right)$ be a real Banach space. With $\|$ $\sigma \|=\max \left\{\left(\left|\sigma_{1}\right|,\left|\sigma_{2}\right|,\left|\sigma_{3}\right|\right)\right\}$, then $(\mathscr{E},\|\|$.$) is a real Banach$ space. Define the partial order $\leq$ on $\mathscr{E}$ by

$$
(\sigma, \xi, v) \geq 0 \Leftrightarrow \sigma \geq 0, \xi \geq 0, v \geq 0 .
$$
in $\mathscr{E}$.

Define $\mathscr{P}=\left\{(\sigma, \xi, v) \in \mathbb{R}^{3}: \sigma, \xi, v \geq 0\right\}$, then, it is a cone

Let $\mathbb{Y}=\{(0,0,0),(1 / 4,0,0),(1 / 4,1 / 4,0),(1 / 4,1 / 4,1 / 4)\}$ $\subset \mathscr{E}$, define order on $\mathbb{Y}$ by $\mathscr{R}=: \leq$ and define $f: \mathbb{Y} \rightarrow \mathbb{Y}$ such that

$f(\sigma)= \begin{cases}(0,0,0) & \text { if } \sigma \in\left\{(0,0,0),\left(\frac{1}{4}, \frac{1}{4}, 0\right),\left(\frac{1}{4}, 0,0\right)\right\}, \\ \left(\frac{1}{4}, \frac{1}{4}, 0\right) & \text { if } \sigma=\left(\frac{1}{4}, \frac{1}{4}, \frac{1}{4}\right) .\end{cases}$

Define the function $d_{c r}: \mathbb{Y} \times \mathbb{Y} \rightarrow \mathscr{E}$ by

$$
d_{c r}\left(\sigma_{1}, \sigma_{2}\right)= \begin{cases}(0,0,0) & \text { if } \sigma_{1}=\sigma_{2}, \\ \left(\frac{1}{4}, \frac{1}{4}, \frac{1}{4}\right) & \text { if } \sigma_{1}, \sigma_{2} \in\left\{(0,0,0),\left(\frac{1}{4}, \frac{1}{4}, 0\right),\left(\frac{1}{4}, \frac{1}{4}, \frac{1}{4}\right)\right\} \\ \left(\frac{1}{4}, 0,0\right) & \text { if } \sigma_{1} \neq \sigma_{2} \text { cannot be both at a time in }\left\{(0,0,0),\left(\frac{1}{4}, \frac{1}{4}, 0\right),\left(\frac{1}{4}, \frac{1}{4}, \frac{1}{4}\right)\right\} .\end{cases}
$$

It is a cone rectangular metric space but not a cone metric space. The assumptions (1)-(4) in Theorem 9 can be verified immediately. Define $T(\sigma)=\sigma / 3$, then, $T \in B(\mathscr{E}, \mathscr{E})$ with $\| T$ $\|_{1} \leq 1$. Now, we verify condition (9). For if $\sigma=(0,0,0)$ and $\kappa=(1 / 4,0,0)$, then

$$
\begin{gathered}
d_{c r}(\sigma, f(\sigma))=d_{c r}(f(\sigma), f(\kappa))=d_{c r}\left(\sigma, f^{2}(\kappa)\right)=(0,0,0), \\
d_{c r}\left(\kappa, f^{2}(\sigma)\right)=\left(\frac{1}{4}, 0,0\right)=d_{c r}(\sigma, \kappa),
\end{gathered}
$$

and thus,

$$
\begin{aligned}
& (I-T)^{2}(I+T) d_{c r}(\sigma, f(\sigma))=(0,0,0), \alpha\left[d_{c r}\left(\sigma, f^{2}(\kappa)\right)+d_{c r}\right. \\
& \cdot\left(\kappa, f^{2}(\sigma)\right]=\alpha\left[(0,0,0)+\left(\frac{1}{4}, 0,0\right)\right]=\alpha\left(\frac{1}{4}, 0,0\right): \alpha \geq 1 .
\end{aligned}
$$

For if $\sigma=(1 / 4,0,0)$ and $\kappa=(1 / 4,1 / 4,0)$, then $d_{c r}(\sigma, f(\sigma$ )$)=d_{c r}(\sigma, \kappa)=d_{c r}\left(\sigma, f^{2}(\kappa)\right)=(1 / 4,0,0) ; d_{c r}\left(\kappa, f^{2}(\sigma)\right)=(1 /$
$4,1 / 4,1 / 4) ; d_{c r}(f(\sigma), f(\kappa))=(0,0,0) ;(I-T)^{2}(I+T)\left(d_{c r}(\right.$ $\sigma, f(\sigma)))=16 / 27(1 / 4,0,0)$ and

$\alpha\left[d_{c r}\left(\sigma, f^{2}(\kappa)\right)+d_{c r}\left(\kappa, f^{2}(\sigma)\right]=\alpha\left[\left(\frac{1}{4}, 0,0\right)+\left(\frac{1}{4}, \frac{1}{4}, \frac{1}{4}\right)\right]=\alpha\left(\frac{2}{4}, \frac{1}{4}, \frac{1}{4}\right)\right.$.

Finally, for $\sigma=(1 / 4,1 / 4,0)$ and $\kappa=(1 / 4,1 / 4,1 / 4)$, we have $d_{c r}(\sigma, f(\sigma))=d_{c r}(f(\sigma), f(\kappa))=d_{c r}\left(\sigma, f^{2}(\kappa)\right)=d_{c r}(\sigma, \kappa)$ $=d_{c r}\left(\kappa, f^{2}(\sigma)\right)=(1 / 4,1 / 4,1 / 4) ;(I-T)^{2}(I+T)\left(d_{c r}(\sigma, f(\sigma)\right.$ )$)=16 / 27(1 / 4,1 / 4,1 / 4)$ and

$\alpha\left[d_{c r}\left(\sigma, f^{2}(\kappa)\right)+d_{c r}\left(\kappa, f^{2}(\sigma)\right]=\alpha\left[\left(\frac{1}{4}, \frac{1}{4}, \frac{1}{4}\right)+\left(\frac{1}{4}, \frac{1}{4}, \frac{1}{4}\right)\right]=\alpha\left(\frac{2}{4}, \frac{2}{4}, \frac{2}{4}\right)\right.$.

Define the function $\mathscr{L}$ by

$$
\begin{aligned}
& \mathscr{L}\left(d_{c r}(f(\sigma), f(\kappa)), d_{c r}(\sigma, \kappa), d_{c r}(\sigma, f(\sigma)), d_{c r}\right. \\
& \left.\quad \cdot(\kappa, f(\kappa)), d_{c r}\left(\sigma, f^{2}(\kappa)\right), d_{c r}\left(\kappa, f^{2}(\sigma)\right)\right)=d_{c r} \\
& \cdot(f(\sigma), f(\kappa))-\alpha\left[d_{c r}\left(\sigma, f^{2}(\kappa)\right)+d_{c r}\left(\kappa, f^{2}(\sigma)\right] .\right.
\end{aligned}
$$


As a consequence, we have $(I-T)^{2}(I+T)\left(d_{c r}(\sigma, f(\sigma))\right.$ $\left.\preceq d_{c r}(\sigma, \kappa)\right)$ implies

$$
\begin{array}{r}
\mathscr{L}\left(d_{c r}(f(\sigma), f(\kappa)), d_{c r}(\sigma, \kappa), d_{c r}(\sigma, f(\sigma)), d_{c r}\right. \\
\left.\cdot(\kappa, f(\kappa)), d_{c r}\left(\sigma, f^{2}(\kappa)\right), d_{c r}\left(\kappa, f^{2}(\sigma)\right)\right) \preceq 0 .
\end{array}
$$

Thus, this example explains Theorem 9 well. Note that $(0,0,0)$ is a fixed point of $f$.

Example 5. Let $\mathscr{E}=C_{R}^{1}[1,2]$ and $\|g\|_{\mathscr{E}}=\|g\|_{\infty}+\left\|g^{\prime}\right\|_{\infty}$, where $\|g\|_{\infty}=\sup _{t \in[1,2]}|g(t)|$ and $\mathscr{P}=\{g(t) \in \mathscr{E}: g(t)>0, t \in[1$, $2]\}$. For each $K \geq 1$, take $g(t)=t$ and $\kappa(t)=t^{2 K}$. By definition $\|g\|=2$ and $\|\kappa\|=2 K+1$. Define the partial order $\leq$ by

for any $g, \kappa \in \mathscr{E} ; g \leq \kappa \Leftrightarrow g(t) \leq \kappa(t) \forall t \in[1,2]$.

Then, $g \leq \kappa$ implies $K\|g\| \leq\|\kappa\|$. Hence, $\mathscr{P}$ is a nonnormal cone. Define $T: \mathscr{E} \rightarrow \mathscr{E}$ such that

$$
(T g)(t)=\frac{1}{2} \int_{1}^{t} g(s) d s
$$

Then, $T \in B(\mathscr{E}, \mathscr{E})$. Let $\mathbb{Y}=\{1,2,3\}$ and $f: \mathbb{Y} \rightarrow \mathbb{Y}$ such that $f(1)=f(2)=1$ and $f(3)=2, f$ is monotone with respect to usual order $(\leq)$ and also assumption (1)-(2) in Theorem 13 are verified. Define $d_{c r}$ by

$$
d_{c r}(\sigma, \varsigma)(t)= \begin{cases}0 & \text { if } \sigma=\varsigma \\ \frac{e^{t}}{3} & \text { if } \sigma, \varsigma \in\{1,2\} \\ e^{t} & \text { otherwise. }\end{cases}
$$

The vector-valued function $d_{c r}$ is a cone rectangular metric space but not a cone metric space. We are left to verify the contractive condition only. For this, if $\sigma=1$ and $\varsigma=2$, then

$$
\begin{gathered}
d_{c r}(\sigma, f(\sigma))=0 ; \\
d_{c r}(f(\sigma), f(\varsigma))=0 ; \\
d_{c r}(\varsigma, f(\varsigma))=\frac{e^{t}}{3},
\end{gathered}
$$

and for $\sigma=2, \varsigma=3$, we have

$$
\begin{gathered}
d_{c r}(\sigma, \varsigma)=e^{t} \\
d_{c r}(\sigma, f(\sigma))=\frac{e^{t}}{3} ; \\
d_{c r}(f(\sigma), f(\varsigma))=\frac{e^{t}}{3} \\
d_{c r}(\varsigma, f(\varsigma))=e^{t}
\end{gathered}
$$

Thus,

$$
\begin{gathered}
(I-T)\left(I-T^{2}\right)\left(d_{c r}(\sigma, f(\sigma))\right)=\frac{e^{t}}{3}\left[1-\frac{t}{2}\left(1-\frac{t^{2}}{4}\right)\right] \\
\left(T d_{c r}(\sigma, \varsigma)\right)(t)=\frac{t e^{t}}{2},\left(T d_{c r}(\varsigma, f(\varsigma))\right)(t)=\frac{t e^{t}}{2}
\end{gathered}
$$

As $t \in[1,2]$ so $t^{2} \leq 4 \Rightarrow t^{2} / 4 \leq 1$ and $1-t^{2} / 4 \geq 0$. Hence,

$$
\begin{aligned}
& (I-T)\left(I-T^{2}\right)\left(d_{c r}(\sigma, f(\sigma))\right) \preceq d_{c r}(\sigma, \varsigma) \text { implies } d_{c r} \\
& \cdot(f(\sigma), f(\varsigma)) \preceq T\left(d_{c r}(\sigma, \varsigma)\right) .
\end{aligned}
$$

Note that 1 is a fixed point of $f$.

\section{A Homotopy Result}

In what follows, we derive a homotopy result by applying Theorem 13.

Theorem 14. Let $(\mathscr{E},\|\|$.$) be a real Banach space and \mathscr{P} \subset \mathscr{E}$ be a cone. Let $\left(\mathbb{Y}, d_{c r}\right)$ be a rectangular cone metric space and $U \subset \mathbb{Y}$ is open. Assume that there exists $T \in B(\mathscr{E}, \mathscr{E})$ and $T($ $\mathscr{P}) \subset \mathscr{P}$. Let the operator $h: \bar{U} \times[0,1] \rightarrow \mathbb{Y}$ satisfy $((39))$ and the condition (1) of Theorem13in the first variable and

(1) $\sigma \neq h(\sigma, \theta)$ for every $\sigma \in \partial U$ ( $\partial U$ denotes the boundary of $U$ in $\mathbb{Y}$ )

(2) there exists $M \geq 0$ such that

$$
\left\|d_{c r}(h(\sigma, \theta), h(\sigma, \mu))\right\| \leq M|\theta-\mu|
$$

for every $\sigma \in \bar{U}$ and $\mu, \theta \in[0,1]$

(3) For some $\sigma \in U$, if there exists $\kappa$ with $\left\|d_{c r}(\sigma, \kappa)\right\| \leq r$, then $\sigma \mathscr{R} \kappa$, where $r$ is radius of open ball in $U$

If $h(\cdot, 0)$ has a fixed point in the open set $U$, then $h(\cdot, 1)$ also has a fixed point in the open set $U$.

Proof. Let

$$
B=\{\theta \in[0,1] \mid \sigma=h(\sigma, \theta) ; \text { for some } \sigma \in U\} .
$$

Define the relation $\preceq$ in $\mathscr{E}$ by $u \preceq v$ if and only if $\|u\| \leq\|v\|$ for all $u, v \in \mathscr{E}$. Next, $0 \in B$, since $h(., 0)$ has a fixed point in the open set $U$. So $B$ is nonempty. Since $d_{c r}(\sigma, h(\sigma, \theta))=d_{c r}$ $(\sigma, \kappa),(I-T)^{2}(I+T)\left(d_{c r}(\sigma, h(\sigma, \theta))\right) \leq d_{c r}(\sigma, \kappa)$ for all $\sigma \mathscr{R}$ $\kappa$, then by Theorem 13 , we have

$$
d_{c r}(h(\sigma, \theta), h(\kappa, \theta)) \leq S\left(d_{c r}(\sigma, \kappa)\right)
$$

Firstly, we show that $B$ is closed in $[0,1]$. For this, let $\left\{\theta_{n}\right\}_{n=1}^{\infty} \subseteq B$ with $\theta_{n} \rightarrow \theta \in[0,1]$ as $n \rightarrow \infty$. It is necessary to prove that $\theta \in B$. Since $\theta_{n} \in B$ for $n \in \mathbb{N}$, there exists $\sigma_{n} \in U$ with $\sigma_{n}=h\left(\sigma_{n}, \theta_{n}\right)$. Since $h(\sigma, \cdot)$ is monotone, so, for $n, m$ 
$\in \mathbb{N}$, we have $\sigma_{m} \mathscr{R} \sigma_{n}$. Since

$$
\begin{gathered}
(I-T)^{2}(I+T)\left(d_{c r}\left(\sigma_{n}, h\left(\sigma_{m}, \theta_{m}\right)\right)\right)=(I-T)^{2} \\
\cdot(I+T)\left(d_{c r}\left(\sigma_{n}, \sigma_{m}\right)\right) \leq d_{c r}\left(\sigma_{n}, \sigma_{m}\right),
\end{gathered}
$$

we have

$$
\begin{aligned}
d_{c r} & \left(h\left(\sigma_{n}, \theta_{m}\right), h\left(\sigma_{m}, \theta_{m}\right)\right) \leq S\left(d_{c r}\left(\sigma_{n}, \sigma_{m}\right)\right), d_{c r} \\
\quad & \left(\sigma_{n}, \sigma_{m+1}\right)=d_{c r}\left(h\left(\sigma_{n}, \theta_{n}\right), h\left(\sigma_{m+1}, \theta_{m+1}\right)\right) \leq d_{c r} \\
\quad & \left(h\left(\sigma_{n}, \theta_{n}\right), h\left(\sigma_{n}, \theta_{m}\right)\right)+d_{c r}\left(h\left(\sigma_{n}, \theta_{m}\right), h\right. \\
\quad & \left.\left(\sigma_{n}, \theta_{m+1}\right)\right)+d_{c r}\left(h\left(\sigma_{n}, \theta_{m+1}\right), h\left(\sigma_{m+1}, \theta_{m+1}\right)\right), \| d_{c r} \\
\quad & \left(\sigma_{n}, \sigma_{m}\right)\left\|\leq M\left|\theta_{n}-\theta_{m}\right|+M\left|\theta_{m}-\theta_{m+1}\right|+\right\| S \\
& \cdot\left(d_{c r}\left(\sigma_{n}, \sigma_{m}\right)\right)\|,\| d_{c r}\left(\sigma_{n}, \sigma_{m}\right) \| \leq \frac{M}{1-\|S\|} \\
& \cdot\left[\left|\theta_{n}-\theta_{m}\right|+\left|\theta_{m}-\theta_{m+1}\right|\right] .
\end{aligned}
$$
have

Since $\left\{\theta_{n}\right\}_{n=1}^{\infty}$ is a RMS Cauchy sequence in $[0,1]$, we

$$
\lim _{n, m \rightarrow \infty} d_{c r}\left(\sigma_{n}, \sigma_{m}\right)=0
$$

that is, $d_{c r}\left(\sigma_{n}, \sigma_{m}\right) \ll c$, whenever $n, m \rightarrow \infty$. Hence, $\left\{\sigma_{n}\right\}$ is a Cauchy sequence in $\mathbb{Y}$. Since $\mathbb{Y}$ is a complete cone rectangular metric space, there exists $\sigma \in \bar{U}$ with $\lim _{n \rightarrow \infty} d_{c r}\left(\sigma_{n}, \sigma\right)$ $\ll c$. Hence, $\sigma_{n} \mathscr{R} \sigma$ for all $n \in \mathbb{N}$. Now consider

$$
\begin{aligned}
& d_{c r}\left(\sigma_{n}, h(\sigma, \theta)\right)=d_{c r}\left(h\left(\sigma_{n}, \theta_{n}\right), h(\sigma, \theta)\right) \leq d_{c r} \\
& \quad \cdot\left(h\left(\sigma_{n}, \theta_{n}\right), h\left(\sigma_{n}, \theta\right)\right)+d_{c r}\left(h\left(\sigma_{n}, \theta\right), h\right. \\
&\left.\quad \cdot\left(\sigma_{n+1}, \theta\right)\right)+d_{c r}\left(h\left(\sigma_{n+1}, \theta\right), h(\sigma, \theta)\right), \| d_{c r} \\
& \quad \cdot\left(\sigma_{n}, h(\sigma, \theta)\right)\left\|\leq M\left|\theta_{n}-\theta\right|+M\left|\theta_{n+1}-\theta\right|+\right\| S \\
& \quad \cdot\left(d_{c r}\left(\sigma_{n+1}, \sigma\right)\right) \| .
\end{aligned}
$$

So we have

$$
\lim _{n \rightarrow \infty} d_{c r}\left(\sigma_{n}, h(\sigma, \theta)\right)=0
$$

Thus, $d_{c r}(\sigma, h(\sigma, \theta))=0$. Hence, $\theta \in B$ and so $B$ is RMS closed in $[0,1]$.

Next, we show that $B$ is open in $[0,1]$. For this, let $\theta_{2} \in B$. Then, we have the existence of $\sigma_{2} \in U$ with $h\left(\sigma_{2}, \theta_{2}\right)=\sigma_{2}$. Since $U$ is open, there exists $r>0$ such that $B\left(\sigma_{2}, r\right) \subseteq U$. Now, assume

$$
l=d_{c r}\left(\sigma_{2}, \partial U\right)=\inf \left\{d_{c r}\left(\sigma_{2}, \xi\right): \xi \in \partial U\right\}
$$

Then, $r=l>0$. Fix $\varepsilon>0$ with $\varepsilon<((1-\|T\|) l) / 2 M$. Let $\theta$ $\epsilon\left(\theta_{1}-\varepsilon, \theta_{1}+\varepsilon\right)$ and $\theta_{1} \in\left(\theta_{2}-\varepsilon, \theta_{2}+\varepsilon\right)$. Then,

$$
\sigma \in B\left(\overline{\sigma_{2}}, r\right)=\left\{\sigma \in X:\left\|d_{c r}\left(\sigma, \sigma_{2}\right)\right\| \leq r\right\} \text {, as } \sigma \mathscr{R} \sigma_{2} .
$$

Consider

$$
\begin{aligned}
d_{c r}\left(h(\sigma, \theta), \sigma_{2}\right)= & d_{c r}\left(h(\sigma, \theta), h\left(\sigma_{2}, \theta_{2}\right)\right) \\
\leq & d_{c r}\left(h(\sigma, \theta), h\left(\sigma, \theta_{1}\right)\right)+d_{c r} \\
& \cdot\left(h\left(\sigma, \theta_{1}\right), h\left(\sigma, \theta_{2}\right)\right)+d_{c r} \\
& \cdot\left(h\left(\sigma, \theta_{2}\right), h\left(\sigma_{2}, \theta_{2}\right)\right), \| d_{c r} \\
& \cdot\left(h(\sigma, \theta), \sigma_{2}\right)\left\|\leq M\left|\theta_{1}-\theta\right|+M\left|\theta_{2}-\theta_{1}\right|+\right\| S \\
& \cdot\left(d_{c r}\left(\sigma_{2}, \sigma\right)\right)\|\leq M \varepsilon+M \varepsilon+\| S\|l=2 M \varepsilon+\| S \| l<l .
\end{aligned}
$$

Thus, for every fixed $\theta \in\left(\theta_{2}-\varepsilon, \theta_{2}+\varepsilon\right), h(\cdot, \theta): B(\bar{\sigma}, r)$ $\rightarrow B(\bar{\sigma}, r)$ has a fixed point in $\bar{U}$ and can be deduced by applying Theorem 13. But this fixed point should be in $U$ as in the previous case. Hence, $\theta \in B$ for any $\theta \in\left(\theta_{2}-\varepsilon, \theta_{2}\right.$ $+\varepsilon)$ and so $B$ is open in $[0,1]$. Thus, we showed that $B$ is RMS open as well as RMS closed in $[0,1]$ and by connectedness, $B=[0,1]$. Hence, $h(\cdot, 1)$ has a fixed point in $U$.

\section{Data Availability}

No data were used to support this study.

\section{Conflicts of Interest}

All authors declare that they have no competing interests.

\section{Authors' Contributions}

All authors contributed equally to this work.

\section{Acknowledgments}

Dr. Sang Og Kim thanks Hallym University Research Fund, 2020 (HRF-202002-017). This work is supported by the Hallym University Research Fund, 2020 (HRF-202002-017).

\section{References}

[1] A. Branciari, "A fixed point theorem of Banach-Caccioppoli type on a class of generalized metric spaces," Publicationes Mathematicae Debrecen, vol. 57, pp. 31-37, 2000.

[2] A. Azam and M. Arshad, "Kannan fixed point theorem on generalized metric spaces," Journal of Nonlinear Sciences and Applications, vol. 1, no. 1, pp. 45-48, 2008.

[3] E. Karapinar, I. M. Erhan, and T. Sekuli, "Fixed points of $(\psi, \phi)$ contractions on rectangular metric spaces," Fixed Point Theory and Applications, vol. 2012, 2012.

[4] C. Di Bari and P. Vetro, "Common fixed points in generalized metric spaces," Applied Mathematics and Computation, vol. 218, no. 13, pp. 7322-7325, 2012.

[5] A. Azam, M. Arshad, and I. Beg, "Banach contraction principle on cone rectangular metric spaces," Applicable Analysis and Discrete Mathematics, vol. 3, no. 2, pp. 236-241, 2009.

[6] R. A. Rashwan, "Some fixed point theorems in cone rectangular metric spaces," Mathematica Aeterna, vol. 2, no. 6, pp. 573587, 2012.

[7] S. Banach, "Sur les opérations dans les ensembles abstraits et leur application aux équations intégrales," Fundamenta Mathematicae, vol. 3, pp. 133-181, 1922. 
[8] J. J. Nieto and R. Rodríguez-López, "Existence and uniqueness of fixed point in partially ordered sets and applications to ordinary differential equations," Acta Mathematica Sinica, vol. 23, no. 12, pp. 2205-2212, 2007.

[9] A. C. M. Ran and M. C. B. Reurings, "A fixed point theorem in partially ordered sets and some applications to matrix equations," Proceedings of American Mathematical Society, vol. 132, pp. 1435-1443, 2004.

[10] P. Debnath, "Fixed points of contractive set valued mappings with set valued domains on a metric space with graph," Turkic $h$ World Mathematical Society Journal of Applied and Engineering Mathematics, vol. 4, no. 2, pp. 169-174, 2014.

[11] J. Jachymski, "The contraction principle for mappings on a metric space with a graph," Proceedings of the American Mathematical Society, vol. 136, no. 4, pp. 1359-1373, 2008.

[12] P. Debnath and M. de La Sen, "Fixed points of eventually $\Delta$ -restrictive and $\Delta(\varepsilon)$-restrictive set-valued maps in metric spaces," Symmetry, vol. 12, no. 1, p. 127, 2020.

[13] S. B. Nadler, "Multivalued contraction mappings," Pacific Journal of Mathematics, vol. 30, no. 2, pp. 475-488, 1969.

[14] M. Neog, P. Debnath, and S. Radenović, "New extension of some common fixed point theorems in complete metric spaces," Fixed Point Theory, vol. 20, no. 2, pp. 567-580, 2019.

[15] B. Samet, C. Vetro, and P. Vetro, "Fixed point theorems for $(\alpha, \psi)$-contractive type mappings," Nonlinear Analysis, vol. 75, no. 4, pp. 2154-2165, 2012.

[16] V. Popa, "Fixed point theorems for implicit contractive mappings," Studii si Cercetari Stiintifice. Serious: Mathematics. Universitatea din Bacau, vol. 7, pp. 127-134, 1997.

[17] V. Popa, "Some fixed point theorems for compatible mappings satisfying an implicit relation," Demonstratio Mathematica, vol. 32, no. 1, pp. 157-163, 1999.

[18] V. Popa, "A general coincidence theorem for compatible multivalued mappings satisfying an implicit relation," Demonstratio Mathematica, vol. 33, pp. 159-164, 1999.

[19] I. Beg and A. R. Butt, "Fixed point for set-valued mappings satisfying an implicit relation in partially ordered metric spaces," Nonlinear Analysis, vol. 71, no. 9, pp. 3699-3704, 2009.

[20] I. Beg and A. R. Butt, "Fixed points for weakly compatible mappings satisfying an implicit relation in partially ordered metric spaces," Carpathian Journal of Mathematics, vol. 25, pp. 1-12, 2009.

[21] V. Berinde, "Stability of Picard iteration for contractive mappings satisfying an implicit relation," Carpathian Journal of Mathematics, vol. 27, pp. 13-23, 2011.

[22] V. Berinde and F. Vetro, "Common fixed points of mappings satisfying implicit contractive conditions," Fixed Point Theory and Applications, vol. 2012, no. 1, 2012.

[23] S. Sedghi, I. Altun, and N. Shobe, "A fixed point theorem for multi-maps satisfying an implicit relation on metric spaces," Appl. Anal. Discrete Math., vol. 2, no. 2, pp. 189-196, 2008.

[24] L. G. Huang and X. Zhang, "Cone metric spaces and fixed point theorems of contractive mappings," Journal of Mathematical Analysis and Applications, vol. 332, no. 2, pp. 14681476, 2007.

[25] S. Rezapour and R. Hamlbarani, "Some notes on the paper "Cone metric spaces and fixed point theorems of contractive mappings"," Journal of Mathematical Analysis and Applications, vol. 345, no. 2, pp. 719-724, 2008.
[26] M. Abbas and B. E. Rhoades, "Fixed and periodic point results in cone metric spaces," Applied Mathematics Letters, vol. 22, no. 4, pp. 511-515, 2009.

[27] M. Nazam, A. Arif, H. Mahmood, and C. Park, "Some results in cone metric spaces with applications in homotopy theory," Open Mathematics, vol. 18, no. 1, pp. 295-306, 2020.

[28] I. Sahin and M. Telci, "Fixed points of contractive mappings on complete cone metric spaces," Hacettepe Journal of Mathematics and Statistics, vol. 38, no. 1, pp. 59-67, 2009.

[29] F. Vetro and S. Radenović, "Some results of Perov type in rectangular cone metric spaces," Journal of Fixed Point Theory and Applications, vol. 20, no. 1, article 41, 2018.

[30] M. Filipović, L. Paunović, S. Radenović, and M. Rajović, "Remarks on "Cone metric spaces and fixed point theorems of T-Kannan and T-Chatterjea contractive mappings"," Mathematical and Computer Modelling bf, vol. 54, no. 5-6, pp. 1467-1472, 2011.

[31] Z. Ercan, "On the end of the cone metric spaces," Topology and its Applications, vol. 166, pp. 10-14, 2014.

[32] Y. Feng and W. Mao, "The equivalence of cone metric spaces and metric spaces," Fixed Point Theory, vol. 11, no. 2, pp. 259-264, 2010.

[33] M. Arshad, J. Ahmad, and E. Karapınar, "Some common fixed point results in rectangular metric spaces," International Journal of Analysis, vol. 2013, Article ID 307234, 7 pages, 2013.

[34] I. Altun, F. Sola, and H. Simsek, "Generalized contractions on partial metric spaces," Topology and its Applications, vol. 157, no. 18 , pp. 2778-2785, 2010.

[35] I. Altun and D. Turkoglu, "Some fixed point theorems for weakly compatible mappings satisfying an implicit relation," Taiwanese Journal of Mathematics, vol. 13, no. 4, pp. 1291$1304,2009$. 